\title{
Cerebral Venous Thrombosis as a Complication of Neuropsychiatric Systemic Lupus Erythematosus
}

\author{
Hiroshi Nishida, Kenji Wakida and Takeo Sakurai
}

\begin{abstract}
A 51-year-old woman undergoing steroid treatment for systemic lupus erythematosus (SLE) was admitted to our hospital after developing a fever, consciousness disturbance and seizures, leading to a diagnosis of SLE-induced meningoencephalitis. Although steroid therapy improved her symptoms, she complained of post-lumbar puncture thunderclap headaches during follow-up, and cerebral venous thrombosis (CVT) was subsequently diagnosed on magnetic resonance venography and cerebral angiography. This is a rare case of neuropsychiatric SLE complicated by CVT during treatment for aseptic meningoencephalitis. The onset of aseptic meningoencephalitis and administration of the lumbar puncture and steroid therapy may have induced the development of the patient's CVT symptoms.
\end{abstract}

Key words: neuropsychiatric SLE, cerebral venous thrombosis, thunderclap headache

(Intern Med 54: 837-841, 2015)

(DOI: 10.2169/internalmedicine.54.3079)

\section{Introduction}

Systemic lupus erythematosus (SLE) may be complicated by a variety of neuropsychiatric symptoms during its clinical course. The neuropsychiatric symptoms of SLE, including peripheral nerve injury, are classified by the American College of Rheumatology as neuropsychiatric SLE (NPSLE) (1). Although previous studies have reported cognitive disorders and headaches as frequently observed symptoms in such patients $(2,3)$, a limited number of case reports have reported the rare complication of cerebral venous thrombosis (CVT) (4-10).

We herein describe a case of SLE accompanied by NPSLE symptoms, including convulsions, acute confusion and aseptic meningoencephalitis, with the subsequent development of CVT associated with post-lumbar puncture thunderclap headaches.

\section{Case Report}

A 51-year-old woman was admitted to our hospital with a fever and consciousness disturbance after a seizure. She had a past history of encephalitis diagnosed at 36 years of age (details unknown), secondary epilepsy as an aftereffect of the encephalitis and SLE. She had been treated with anticonvulsants (phenytoin, $240 \mathrm{mg} /$ day; valproic acid, $800 \mathrm{mg} /$ day) and low-dose corticosteroids (prednisolone, $10 \mathrm{mg} /$ day) for SLE at another hospital. She subsequently developed a fever the day before admission to our hospital, followed by tonic convulsions lasting approximately one minute with a consciousness disturbance and fever on the day of admission.

Upon admission, the patient's vital signs were as follows: body temperature, $38^{\circ} \mathrm{C}$; blood pressure, $102 / 56 \mathrm{mmHg}$; pulse rate, 98 beats/min; and consciousness level according to the Japan Coma Scale, 3. No abnormal findings were observed on cardiovascular, respiratory or abdominal examinations. A neurological examination revealed mild neck rigidity, with no other focal neurological deficits.

A complete blood cell count demonstrated the following normal findings: white blood cells, $8,600 / \mu \mathrm{L}$; red blood cells, $384 \times 10^{4} / \mu \mathrm{L}$; and platelets, $24 \times 10^{4} / \mu \mathrm{L}$. Blood biochemistry showed an elevated C-reactive protein level and erythrocyte sedimentation rate $(19 \mathrm{mg} / \mathrm{dL}$ and $58 \mathrm{~mm} / \mathrm{h}$, respectively), with a PT of $80.5 \%$ (normal, 70-130). Serology was positive for antinuclear antibodies [1:320 (normal, $<40$ fold)] and negative for anti-ds-DNA IgG [10 IU/mL (nor- 


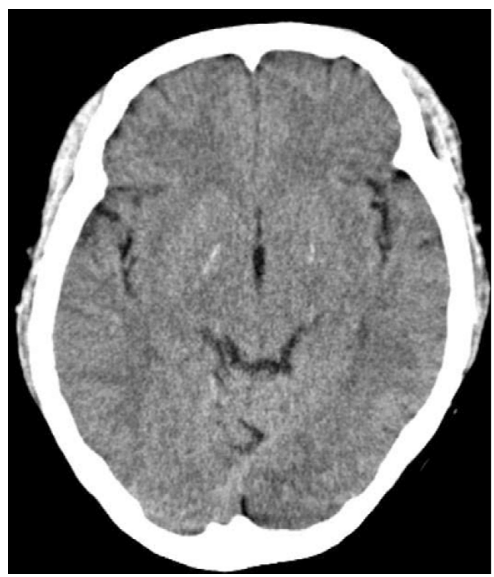

(a)

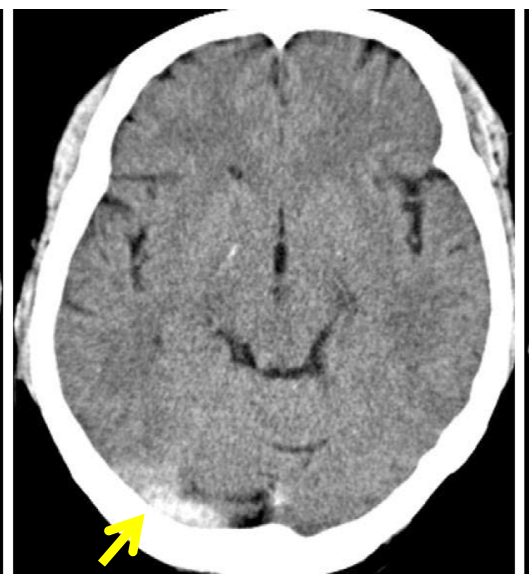

(b)

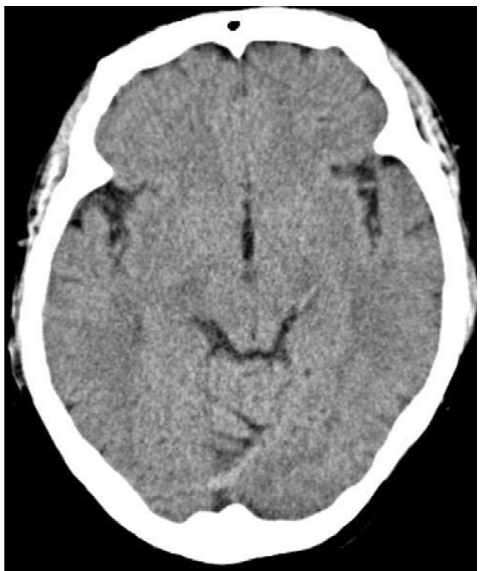

(c)

Figure 1. Brain computed tomography (CT) performed upon the appearance of symptoms (b) demonstrated a high-density area in the right transverse sinus (arrow), leading to a suspected diagnosis of cerebral venous thrombosis. Brain CT performed on admission (a) and follow-up (c) showed no abnormalities.

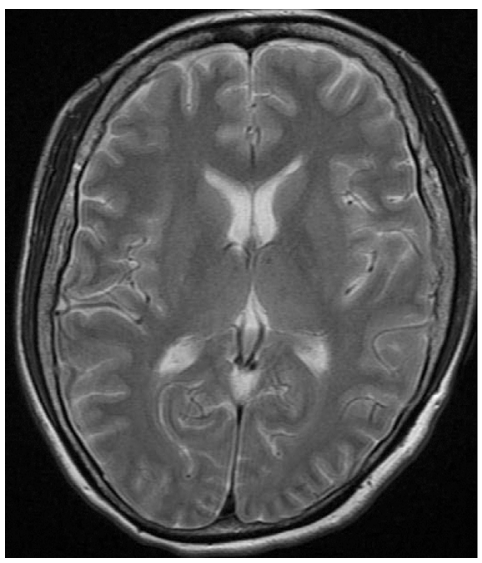

(a)

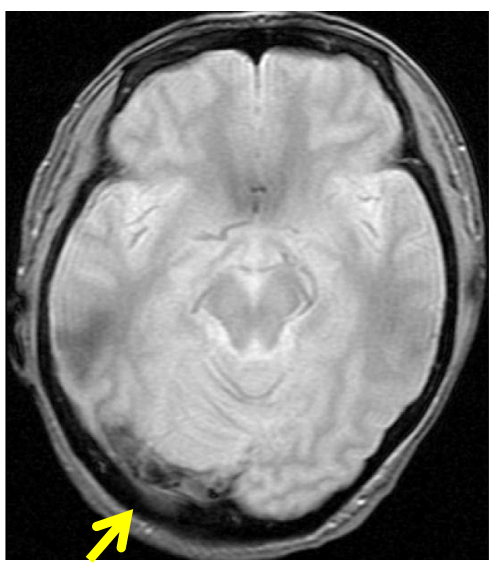

(b)

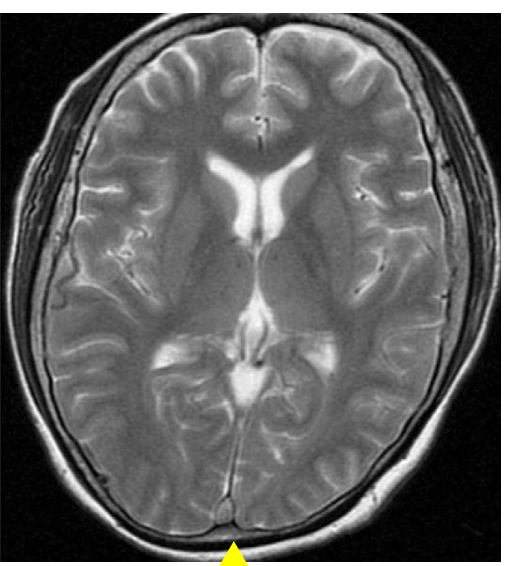

(c)

Figure 2. A brain magnetic resonance imaging (MRI) T2*-weighted image (b) showed a low-intensity area in the right transverse sinus (arrow), and a diagnosis of transverse sinus thrombosis was suspected. Thrombosis was subsequently detected in the superior sagittal sinus as an area with an iso-intense signal on a brain MRI T2-weighted image (arrow) (c). A brain MRI T2-weighted image obtained on admission (a) showed no abnormalities.

mal, $\leq 12 \mathrm{IU} / \mathrm{mL}$ )], anti-ds-DNA IgM $[2 \mathrm{U} / \mathrm{mL}$ (normal, 6 $\mathrm{IU} / \mathrm{mL}$ )], anti-Sm antibodies, lupus anticoagulant $[1.02$ (normal, <1.3)], anticardiolipin antibody IgG $[8 \mathrm{U} / \mathrm{mL}$ (normal, $<10 \mathrm{U} / \mathrm{mL})]$ and anticardiolipin antibody $\operatorname{IgM}[5 \mathrm{U} / \mathrm{mL}$ (normal, $<8 \mathrm{U} / \mathrm{mL}$ )]. The D-dimer level was not measured.

A lumbar puncture revealed a primary pressure of 100 $\mathrm{mm} \mathrm{H}_{2} \mathrm{O}$, cell count of $349 / 3 \mu \mathrm{L}(\mathrm{N} / \mathrm{L}=7 / 342)$, protein level of $271 \mathrm{mg} / \mathrm{dL}$, glucose level of $54 \mathrm{mg} / \mathrm{dL}$, myelin basic protein level of $40 \mathrm{pg} / \mathrm{mL}$, elevated interleukin-6 (IL-6) level of $331 \mathrm{pg} / \mathrm{mL}$, the absence of bacteria and tuberculosis microorganisms and negative findings for herpes simplex virus polymerase chain reaction, varicella-zoster virus polymerase chain reaction and oligoclonal bands. Brain computed to- mography (CT) and magnetic resonance imaging (MRI) showed no abnormalities (Fig. 1, 2).

Although the patient received acyclovir (1,500 mg/day) and antibiotics for aseptic meningoencephalitis, she did not respond to the treatment. A diagnosis of aseptic meningoencephalitis as a complication of SLE was thus made based on the pre-existing SLE and elevated IL-6 level in the spinal fluid. Steroid treatment was administered (prednisolone, 60 $\mathrm{mg} /$ day), and her symptoms quickly improved on day 6 .

On day 11, a repeated lumbar puncture showed improvements in the data, with a cell count of $44 / 3 \mu \mathrm{L}$, protein level of $56 \mathrm{mg} / \mathrm{dL}$ and IL-6 level of $33 \mathrm{pg} / \mathrm{mL}$. However, the patient suddenly developed a headache two hours after the 


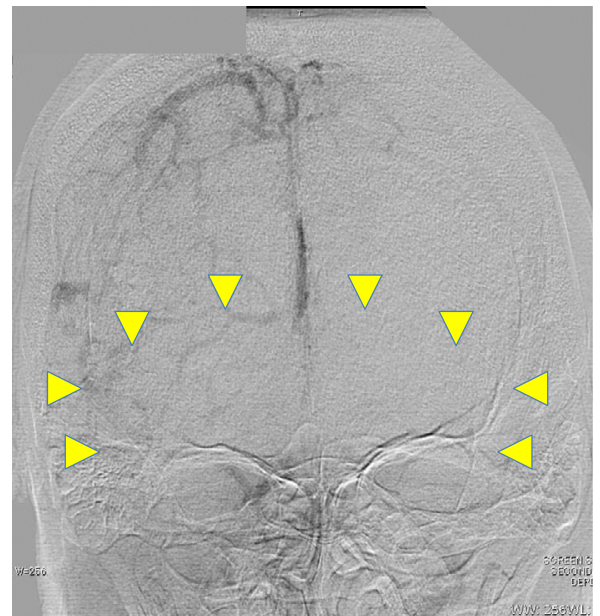

(a)

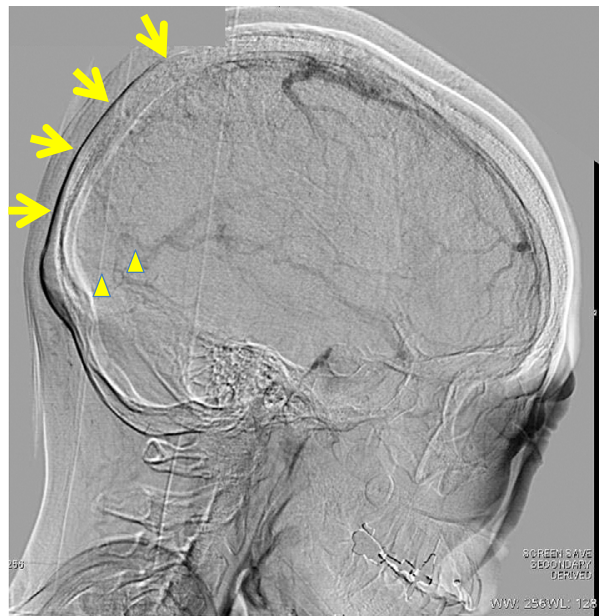

(b)

Figure 3. A digital subtraction cerebral angiogram of the right carotid artery (venous phase) (a: frontal view, b: lateral view) did not visualize the superior sagittal sinus (b: arrows), straight sinus (b: arrowheads), bilateral transverse sinus (a: arrowheads) or sigmoid sinus (a: arrowheads); cerebral venous thrombosis was diagnosed.

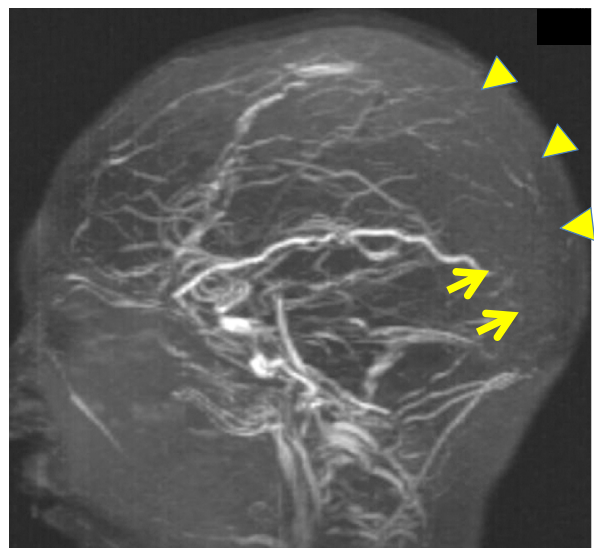

(a)

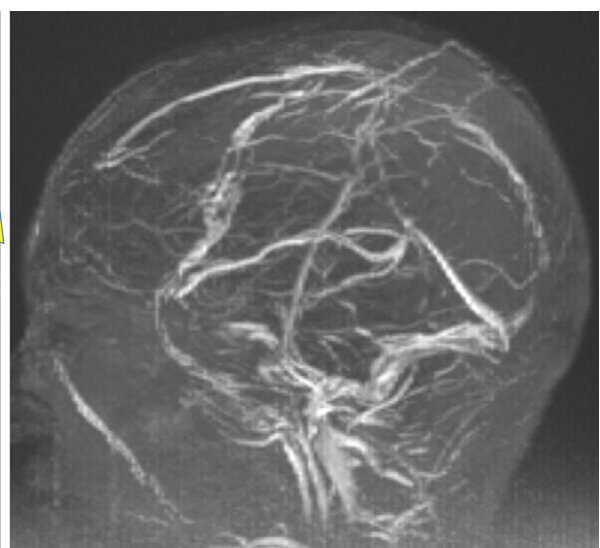

(b)

Figure 4. Magnetic resonance venography (MRV) performed upon the appearance of symptoms (a) did not visualize the superior sagittal sinus (arrowheads) or straight sinus (arrows), cerebral venous thrombosis was diagnosed. MRV performed six months later (b) successfully visualized the superior sagittal sinus, transverse sinus and straight sinus; the site of cerebral venous thrombosis had been recanalized.

lumbar puncture that immediately reached a peak in severity (similar to a thunderclap headache). Although an analgesic was administered, the headache was not relieved. In addition, the presence of a high-density area in the right transverse sinus on brain CT (Fig. 1) was suspicious of venous sinus thrombosis. However, no brain lesions were detected, such as cerebral hemorrhage. Nevertheless, an MRI T2*weighted image showed low intensity in the right transverse sinus, and a diagnosis of transverse sinus thrombosis was suspected. Thrombosis was subsequently detected in the superior sagittal sinus based on an iso-intense signal on a brain MRI T2-weighted image (Fig. 2).

Digital subtraction cerebral angiography (DSA) (Fig. 3) and MR venography (MRV) (Fig. 4) showed occlusion of the superior sagittal sinus, straight sinus, bilateral transverse sinus and sigmoid sinus, confirming the diagnosis of CVT. A blood test showed elevation of the D-dimer level $(5.4 \mu \mathrm{g} /$ $\mathrm{mL}$ ). Anti-coagulation therapy with heparin-Na was therefore started and subsequently changed to warfarin. No complications involving the brain parenchyma, such as cerebral hemorrhage, were observed after treatment, and the patient's clinical course was uneventful, including the alleviation of her headache. Follow-up MRV (Fig. 4) showed recanalization of the venous sinus. 
Table. Reported of the Cases of Crebral Venous Thrombosis in Systemic Lupus Erthematosis (Negative Antiphospholipid Antibodies Cases)

\begin{tabular}{ccccccc}
\hline Reference & Age & Sex & renal disorder & APLA & Treatment & Outcome \\
\hline 5 & 40 & F & - & - & heparin, wafarin & Resolved \\
7 & 34 & F & + & - & steroid pulse, heparin, warfarin & Resolved \\
7 & 55 & F & + & - & steroid pulse, heparin, warfarin & Resolved \\
8 & 14 & F & - & - & heparin, steroid, wafarin & Resolved \\
8 & 13 & F & - & - & steroid & Resolved \\
9 & 39 & F & - & - & steroid, cyclophosphamide, anticoagulant & Resolved \\
10 & 33 & F & - & - & steroid & Resolved \\
our case & 51 & F & - & - & steroid, anticoagulant & \\
\multicolumn{7}{l}{ APLA: anti-phospholipid antibody (lupus anticoagulant and anticardiolipin antibody), } \\
+: positive, -: negative
\end{tabular}

\section{Discussion}

The present case report involves a patient who developed a variety of neuropsychiatric symptoms, including convulsions, acute confusion and aseptic meningoencephalitis, during the clinical course of SLE. Moreover, CVT was diagnosed following the onset of a post-lumbar puncture headache.

Previous case reports have reported complication rates of convulsions, acute confusion and meningitis, neuropsychiatric symptoms associated with SLE collectively known as NPSLE, of $9-16 \%, 7 \%$ and $2 \%$, respectively $(2,3)$. However, no cases of CVT have been described in previous reports, and this symptom is thus considered to be a rare complication.

Although the Virchow triad (a hypercoagulable state, blood flow congestion and structural changes in the vascular wall) is thought to play a role in the onset of CVT, several other causes of this complication are suspected. Acquired causes include infections, such as meningoencephalitis and otitis media, brain tumors, pregnancy and puerperium, oral contraceptive use and surgery, while hereditary causes comprise protein $\mathrm{C}$ and $\mathrm{S}$ deficiencies. However, SLE accounts for only $1 \%$ of underlying causes of CVT $(11,12)$.

Although the development of CVT during the clinical course of SLE is rare, some cases have been shown to involve anti-phospholipid antibodies (4-6), while others were negative for anti-phospholipid antibodies, with suspected underlying causes of hypercoagulability, vasculitis and hypertrophic pachymeningitis (5, 7-10) (Table).

The present report discusses a case of CVT that occurred after lumbar puncture. Previous reports have suggested that lumbar puncture accounts for $1.9 \%$ of all causes of CVT (10), possibly attributable to a reduced amount of cerebrospinal fluid. Other suspected causes of CVT include venous dilatation resulting from cerebrospinal fluid reduction and associated decreases in the venous blood flow, increased venous blood viscosity, stenosis and occlusion of the veins and venous sinuses due to cerebral ptosis $(13,14)$. In addition, the administration of drugs, such as steroids, may promote the development of CVT after lumbar puncture $(15,16)$, with lumbar puncture and corticosteroid treat- ment likely to play a role in the onset of CVT in patients with multiple sclerosis (17-19).

In the current case, the aseptic meningoencephalitis appeared to be a symptom of NPSLE. Moreover, the initially elevated IL-6 level decreased following treatment with corticosteroids.

In patients with NPSLE, the CSF levels of IL-6 and IL-8 are initially elevated and subsequently decrease after successful treatment. Furthermore, analyses of the CSF cytokine levels, particularly IL-6 and IL-8, are useful for making the diagnosis and possibly in providing follow-up in patients with NPSLE (20). Hence, intrathecal immunological inflammation plays an important role in the pathogenesis of NPSLE (21). In this case, anti-phospholipid antibodies were negative and not associated with anti-phospholipid antibodies. Therefore, the direct expansion of inflammation due to meningoencephalitis and the administration of steroid therapy may have induced the development of CVT. Moreover, the lumbar puncture was associated with the onset of the CVT in association with a thunderclap headache.

Taken together, we conclude that the direct expansion of inflammation resulting from aseptic meningoencephalitis and the administration of the lumbar puncture and steroid treatment with prednisolone played a role in the onset of CVT in the present case.

Lastly, the patient's severe post-lumbar puncture headache appeared suddenly, with the severity peaking immediately. Even with the administration of an analgesic, the headache persisted until the following day and resembled the features of a thunderclap headache. The classic symptom of CVT is headache, observed in approximately $90 \%$ of cases (11). In addition, Cumurciuc et al. reported that headaches are observed as the only symptom of CVT in $14 \%$ of cases, with thunderclap headaches in $17.5 \%$ of cases as the only symptom. It has been reported that a diagnosis of CVT should be ruled out on MRI and/or MRV in cases of disease progression with a thunderclap headache, even if CT and spinal tap examinations show normal findings (22). Although headache is a common disease manifestation, CVT must be excluded in patients exhibiting thunderclap headaches and/or progressive deterioration.

We herein reported the case of a patient who developed a variety of neuropsychiatric symptoms, including convul- 
sions, acute confusion and meningoencephalitis. Although steroid treatment successfully improved these symptoms, CVT was ultimately diagnosed because the patient developed a thunderclap headache after undergoing lumbar puncture. While a variety of causes may have contributed to the onset of CVT in the present case, the lumbar puncture was likely an aggravating factor. Our findings suggest that the risk of CVT is increased in the context of pre-existing disease and treatment requiring lumbar puncture, a relatively common examination in daily practice in the field of neurological medicine. Therefore, careful follow-up after lumbar puncture is warranted.

\section{The authors state that they have no Conflict of Interest (COI).}

\section{References}

1. ACR Ad Hoc Committee on Neuropsychiatric Lupus Nomenclature. The American college of rheumatology nomenclature and case definitions for neuropsychiatric lupus syndromes. Arthritis Rheum 42: 599-608, 1999.

2. Ainiala H, Loukkola J, Peltola J, Korpela M, Hietaharji A. The prevalence of neuropsychiatric syndromes in systemic lupus erythematosus. Neurology 57: 496-500, 2001.

3. Brey RL, Holliday SL, Saklad AR, et al. Neuropsychiatric syndromes in lupus. prevalence using standard definitions. Neurology 58: 1214-1220, 2002

4. Vidailhet M, Piette JC, Wechsler B, Bousser MG, Brunet P. Cerebral venous thrombosis in systemic lupus erythematosus. Stroke 21: 1226-1231, 1990

5. Flusser D, Abu-Shakra M, Baumgarten-Kleiner A, Flusser G, Sukenik S. Superior sagittal sinus thrombosis in a patient with systemic lupus erythematosus. Lupus 5: 334-336, 1996.

6. Carhuapoma JR, Mitsias P, Levine SR. Cerebral venous thrombosis and anticardiolipin antibodies. Stroke 28: 2363-2369, 1997.

7. Lee MK, Kim JH, Kang HR, et al. Systemic lupus erythematosus complicated with cerebral venous sinus thrombosis: a report of two cases. J Korean Med Sci 16: 351-354, 2001.

8. Cruz BA, Santos LA, Damasceno RP, et al. Dural sinus thrombosis in childhood systemic lupus erythematosus. J Rheumatol 28 : 2140-2141, 2001.

9. Takashima T, Koarada S, Mitamura M, et al. A case of CNS lupus complicated by right transverse sinus thrombosis. Kyushu Ryumachi (Kyushu Journal of Rheumatology) 25: 49-54, 2005 (in Japanese, Abstract in English).

10. Ohsaka M, Nonaka T, Oka S, Minamida Y, Mikami T. Superior sagittal sinus thrombosis in a case of longstanding systemic lupus erythematosus. No To Shinkei (Brain and Nerve) 58: 57-61, 2006 (in Japanese, Abstract in English).

11. Ferro JM, Canhão P, Stam J, Bousser MG, Barinagarrementeria F; ISCVT Investigators. Prognosis of cerebral vein and dural sinus thrombosis: results of the International Study on Cerebral Vein and Dural Sinus Thrombosis (ISCVT). Stroke 35: 664-670, 2004.

12. Saposnik G, Barinagarrementeria F, Brown RD Jr, et al; American Heart Association Stroke Council and the Council on Epidemiology and Prevention. Diagnosis and management of cerebral venous thrombosis: a statement for healthcare professionals from the American Heart Association/American Stroke Association. Stroke 42: 1158-1192, 2011.

13. Canhão $P$, Batista $P$, Falcão $F$. Lumbar puncture and dural sinus thrombosis: a causal or casual association? Cerebrovasc Dis 19: 53-56, 2005.

14. Milhaud D, Heroum C, Charif M, Saulnier P, Pages M, Blard JM. Dural puncture and corticotherapy as risks factors for cerebral venous sinus thrombosis. Eur J Neurol 7: 123-124, 2000.

15. Benzon HT, Iqbal M, Tallman MS, Boehlke L, Russell EJ. Superior sagittal sinus thrombosis in a patient with postdural puncture headache. Reg Anesth Pain Med 28: 64-67, 2003.

16. Aidi S, Chaunu MP, Biousse V, Bousser MG. Changing pattern of headache pointing to cerebral venous thrombosis after lumbar puncture and intravenous high-dose corticosteroids. Headache 39: 559-564, 1999.

17. Vandenberghe N, Debouverie M, Anxionnat R, Clavelou P, Bouly $\mathrm{S}$, Weber M. Cerebral venous thrombosis in four patients with multiple sclerosis. Eur J Neurol 10: 63-66, 2003.

18. Maurelli M, Bergamaschi R, Candeloro E, Todeschini A, Micieli G. Cerebral venous thrombosis and demyelinating diseases: report of a case in a clinically isolated syndrome suggestive of multiple sclerosis onset and review of the literature. Mult Scler 11: 242244, 2005.

19. Presicci A, Garofoli V, Simone M, Campa MG, Lamanna AL, Margari L. Cerebral venous thrombosis after lumbar puncture and intravenous high dose corticosteroids: a case report of a childhood multiple sclerosis. Brain Dev 35: 602-605, 2013.

20. Carlsten H, Tarkowski A. Intrathecal cytokines in systemic lupus erythematosus with central nervous system involvement. Lupus 9: 498-503, 2000.

21. Kato T, Shiratori K, Kobashigawa T, Hidaka Y. Systemic lupus erythematosus with organic brain syndrome: serial electroencephalograms accurately evaluate therapeutic efficacy. Intern Med 45: 95-99, 2006.

22. Cumurciuc R, Crassard I, Sarov M, Valade D, Bousser MG. Headache as the only neurological sign of cerebral venous thrombosis: a series of 17 cases. J Neurol Neurosurg Psychiatry 76: 10841087, 2005.

(C) 2015 The Japanese Society of Internal Medicine http://www.naika.or.jp/imonline/index.html 